\title{
COSMIC-RAY ACCELERATION EFFICIENCY VERSUS TEMPERATURE EQUILIBRATION: THE CASE OF SNR 0509-67.5
}

\author{
E. A. Helder, D. KosenKo, And J. Vink \\ Astronomical Institute, Utrecht University, P.O. Box 80000, NL-3508 TA Utrecht, The Netherlands; e.a.helder@ astro-uu.nl \\ Received 2010 May 14; accepted 2010 July 12; published 2010 July 28
}

\begin{abstract}
We study the 0509-67.5 supernova remnant in the Large Magellanic Cloud with the VLT/FORS2 spectrograph. We detect a broad component in the $\mathrm{H} \alpha$ emission with an FWHM of $2680 \pm 70 \mathrm{~km} \mathrm{~s}^{-1}$ and $3900 \pm 800 \mathrm{~km} \mathrm{~s}^{-1}$ for the southwest (SW) and northeast (NE) shocks, respectively. For the SW, the proton temperature appears to be too low for the shock velocity, which we attribute to a cosmic-ray pressure behind the shock front of at least $20 \%$ of the total pressure. For the NE, the post-shock proton temperature and the shock velocity are compatible, only if the plasma behind the shock front has a degree of thermal equilibrium of over $20 \%$, which is at odds with current models for temperature equilibration behind fast shocks, which do not accelerate cosmic rays. If we assume the electron temperature to be less than $10 \%$ of the proton temperature, we find a post-shock cosmic-ray pressure of at least $7 \%$.
\end{abstract}

Key words: acceleration of particles - ISM: individual objects (SNR 0509-67.5) - ISM: supernova remnants radiation mechanisms: thermal - shock waves

\section{INTRODUCTION}

Supernova remnants (SNRs) are generally thought to be the dominant sources of Galactic cosmic rays. For this to be true, SNRs need to transfer about $10 \%$ of their initial kinetic energy into cosmic rays. An open question is whether SNR shocks can reach these acceleration efficiencies averaged over their life time; recent $\mathrm{TeV}$ and $\mathrm{GeV} \gamma$-ray observations give promising but ambiguous results (Ellison et al. 2010; Abdo et al. 2010).

Although the process of efficiently accelerating particles is well understood (Malkov \& Drury 2001), observational verifications for efficient acceleration are scarce (e.g., Warren et al. 2005; Lee et al. 2007; Vink et al. 2006; Helder et al. 2009). These observations are essential for characterizing the efficiency of the acceleration. As the particles move ahead (i.e., upstream) of the shock, they form a so-called shock precursor, which compresses and pre-heats the upstream medium. This effectively lowers the Mach number of the main shock, thereby lowering the temperature behind the shock front (Ellison et al. 2004; Drury et al. 2009). This effect would ideally be characterized by the post-shock proton temperature $\left(T_{\mathrm{s}, \mathrm{p}}\right)$, as $T_{\mathrm{s}, \mathrm{p}}$ is close to the plasma temperature, whereas the post-shock electron temperature $T_{\mathrm{s}, \mathrm{e}}$ might be lower (e.g., Ghavamian et al. 2007b). The latter implies that the electrons might constitute only a minor part of the thermal pressure behind the shock front.

For some SNRs, $T_{\mathrm{s}, \mathrm{p}}$ can be determined from hydrogen line emission at the shock fronts; these are so-called Balmerdominated shocks. The hydrogen lines of a Balmer-dominated shock consist of two superimposed components: the narrow component emitted by neutral hydrogen after entering the shock front and the broad component by hot protons after undergoing charge exchange with incoming neutral hydrogen atoms. The width of the broad component reflects the proton temperature behind the shock front (Chevalier et al. 1980; Heng 2009).

In this Letter, we report on our study of SNR 0509-67.5, as this is a likely source of efficient cosmic-ray acceleration; recent studies show that the remnant resulted from a highly energetic Type Ia explosion (Hughes et al. 1995; Badenes et al. 2008; Rest et al. 2008). Its X-ray spectrum provides some evidence for non-thermal emission (Warren \& Hughes 2004). In addition, hydrodynamical models show that the presence of highly energetic particles is likely not negligible (Kosenko et al. 2008).

SNR 0509-67.5 was discovered to have Balmer-dominated shocks by Tuohy et al. (1982), although the flux in the broad component was too low to be detected. A subsequent attempt by Smith et al. (1991) did not reveal any broad component either. The first detection of a broad component in the hydrogen line emission of this remnant was by Ghavamian et al. (2007a), who measured the width of the broad component of the $\operatorname{Ly} \beta$ line to be $3700 \pm 400 \mathrm{~km} \mathrm{~s}^{-1}$, corresponding to a shock velocity $\left(v_{\mathrm{s}}\right)$ of $5200-6300 \mathrm{~km} \mathrm{~s}^{-1}$. However, as the spectrum was taken from the entire remnant, it remains uncertain whether the obtained line width is broadened by the bulk motion of the plasma as well.

We report the detection of a broad component in the $\mathrm{H} \alpha$ line emission of SNR 0509-67.5 at two locations of the shock front. We combine this with a shock velocity based on X-ray observations (Kosenko et al. 2008) to determine the fraction of the post-shock pressure contributed by cosmic rays.

\section{OPTICAL DATA AND RESULTS}

We observed SNR 0509-67.5 for 10932 s (four blocks of 2733 s) with FORS2, the low-dispersion spectrograph of ESO's VLT (Appenzeller et al. 1998). The observations were made on 2009 October 15 and 20 and November 10 and 11 . By centering the slit at a bright star at $\alpha=05: 09: 28.793, \delta=-67: 31: 30.83$ (J2000), with a position angle of $42^{\circ}$ (Figure 1), we obtained spectra of both the SW and NE rims with a single pointing. The width of the slit was 1".6 which, in combination with the 600RI grism and the $2 \times 2$ binned readout, corresponds to a resolution of $\sim 485 \mathrm{~km} \mathrm{~s}^{-1}$ at $\mathrm{H} \alpha(6563 \AA)$. This resolution prevents us from resolving the narrow component of the $\mathrm{H} \alpha$ line, but it increases the signal to noise sufficiently to detect and resolve the broad component. The data were corrected for bias, flat fielded, and the skylines and cosmic rays were removed. The wavelength calibration was done by fitting a fourth order polynomial to the spectral lines of $\mathrm{He}, \mathrm{HgCd}, \mathrm{Ar}$, and $\mathrm{Ne}$ lamps, obtained during daytime. In addition, we checked our calibration against the position of three skylines (at 5577.3, 6864.0, and 7571.7 $\AA$, respectively; Osterbrock et al. 1996). The absolute wavelength 

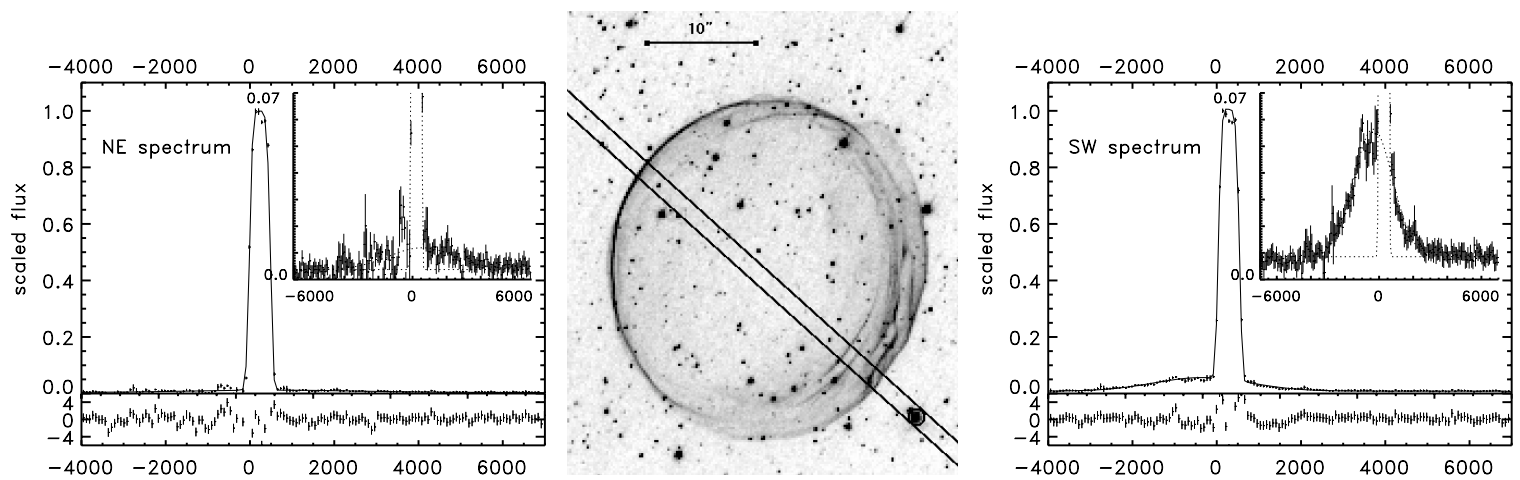

Figure 1. Left: spectrum and best fit of the NE shock of SNR 0509-67.5. Middle: archival HST/ACS observation of SNR 0509-67.5, as obtained at 2006 October 28 , with an exposure time of $4620 \mathrm{~s}$. The position of the long slit of the VLT/FORS2 instrument is indicated. The star on which the slit is centered is indicated with a circle. North is up, east is to the left. Right: spectrum and best fit of the SW shock. The lower panels show the residuals divided by the errors. The insets show a zoom-in of the broad component, with the best-fit narrow and broad components overplotted.

Table 1

Best-fit Parameters for the H $\alpha$ Lines of both the NE and SW Spectra

\begin{tabular}{|c|c|c|}
\hline Parameter & SW & $\mathrm{NE}$ \\
\hline Center narrow $\left(\mathrm{km} \mathrm{s}^{-1}\right)$ & $287.0 \pm 1.4$ & $286.0 \pm 1.5$ \\
\hline Center broad $\left(\mathrm{km} \mathrm{s}^{-1}\right)$ & $-342 \pm 28$ & $459 \pm 220$ \\
\hline FWHM broad $\left(\mathrm{km} \mathrm{s}^{-1}\right)$ & $2680 \pm 70$ & $3900 \pm 800$ \\
\hline Total flux ${ }^{\mathrm{a}}\left(10^{-16} \mathrm{erg} \mathrm{s}^{-1} \mathrm{~cm}^{-2} \operatorname{arcsec}^{-2}\right)$ & 8.6 & 5.3 \\
\hline$I_{\mathrm{b}} / I_{\mathrm{n}}$ & $0.29 \pm 0.01$ & $0.08 \pm 0.02$ \\
\hline
\end{tabular}

Notes. ${ }^{\text {a }}$ Approximate flux calibration based on an observation of a photometric standard star (LTT 2415; Hamuy et al. 1994) taken on 2010 November 11.

calibration has a systematic uncertainty of $0.5 \operatorname{pixel}\left(37 \mathrm{~km} \mathrm{~s}^{-1}\right)$ and the errors on the data points were determined by tracing the error propagation through the reduction steps, starting from the raw data. For each observation block, obvious outliers, probably caused by erroneous skyline subtraction, were removed. The resulting spectra were fitted with two superimposed Gaussians and an offset, convolved with the slit width. The best-fitting parameters for both spectra are listed in Table 1. In order to determine the significance of the broad component in the NE spectrum, we compared the obtained $\chi^{2}$ value (464) with a fit in which we only fitted a single Gaussian to the spectrum (525). The difference indicates a significance of $7.8 \sigma$. The $\chi_{\text {red }}^{2}$ values are 2.03 and 2.56 for the SW and NE fits, respectively. These high values are mainly caused by the substructure of the narrow line: a fit to the broad component with a single Gaussian, excluding the central region (between -100 and $800 \mathrm{~km} \mathrm{~s}^{-1}$ ), convolved with the resolution, gives $\chi_{\text {red }}^{2}$ of 0.51 for the SW and 0.76 for the NE. This substructure might either be caused by spatial surface brightness fluctuations of the remnant within the slit, or by errors in the skyline subtraction. We determine the $1 \sigma$ errors on the parameters for the broad components by using the errors we determined in our data reduction. For the narrow component we first increased the errors such that $\chi_{\text {red }}^{2}=1$, and then determined the $1 \sigma$ errors, using $\Delta \chi^{2}=1$.

\section{COMPARING WITH SHOCK VELOCITIES}

To determine the shock velocity, we use the X-ray line width of $\sigma_{\mathrm{RGS}}=4900 \pm 420 \mathrm{~km} \mathrm{~s}^{-1}$, as observed by the Reflection Grating Spectrometer (RGS) on board XMM-Newton (Kosenko et al. 2008). This line width is caused by both the thermal and bulk broadening of the plasma. We follow the method of Kosenko et al. (2010) to disentangle the bulk broadening from the thermal broadening to obtain the forward shock velocity, as described below.

As shown in Kosenko et al. (2010), $\sigma_{\mathrm{RGS}}$ and $v_{\mathrm{S}}$ are related as follows:

$$
\sigma_{\mathrm{RGS}}=\left(v_{\mathrm{s}} / 4\right) \sqrt{3 r_{\mathrm{sh}}^{2}+9 r_{\text {bulk }}^{2}},
$$

where $r_{\mathrm{sh}}=v_{\mathrm{rs}} / v_{\mathrm{s}}$ is the ratio of the reverse shock velocity to the forward shock velocity and $r_{\text {bulk }}=v_{\text {bulk, ej }} / v_{\text {bulk, CSM }}$ is the gradient in the plasma bulk velocity from reverse to forward shock. We obtain $r_{\mathrm{sh}} \simeq 1$ (whole remnant) and $r_{\mathrm{sh}} \simeq 0.5(\mathrm{NE})$, from analytical models (Truelove \& McKee $1999)$ with $M_{\mathrm{ej}}=1.4 M_{\odot}, E=1.4 \times 10^{51} \mathrm{erg}$ and an age of 400 years (Badenes et al. 2008; Rest et al. 2008). Additionally, we constrain the models with a forward shock radius of $15^{\prime \prime} \cdot 9 \pm 00^{\prime \prime} 8$ and $16^{\prime \prime} .3 \pm 00^{\prime} 3$ for the entire remnant and the NE, respectively, and a reverse shock radius of $11^{\prime \prime} .4 \pm 2$ ". 1 and $13 . .5 \pm 0$ '.2 for the entire remnant and the NE, respectively, based on a deprojection of Chandra images (48.9 ks, ObsID: 776), following the procedure of Kosenko et al. (2010).

We obtain $r_{\text {bulk }}=0.95$ with numerical simulations (Sorokina et al. 2004; Kosenko 2006), using the above parameters.

We use Equation (1) to estimate $v_{\mathrm{s}}=6000 \pm 300 \mathrm{~km} \mathrm{~s}^{-1}$ (whole remnant) and $v_{\mathrm{s}}=6600 \pm 400 \mathrm{~km} \mathrm{~s}^{-1}$ (NE). In the remainder of this paper, we use conservative $v_{\mathrm{s}}$ estimates of $5000 \mathrm{~km} \mathrm{~s}^{-1}$ for the SW and $6000 \mathrm{~km} \mathrm{~s}^{-1}$ for the NE.

To check these shock velocities, we estimated the expansion with Chandra. Chandra observed SNR 0509-67.5 for three times, with the first observation in 2000 May (earlier used in this section to determine forward and reverse shock radii) and the latter two in 2007 May (29.5 and $32.7 \mathrm{ks}$; ObsIDs 7635 and 8554). Following the method of Vink (2008b), we find a shock velocity of $6700 \pm 400 \mathrm{~km} \mathrm{~s}^{-1}$ averaged over the azimuth of the remnant. Note that a full proper motion study of SNR 0509-67.5, using both Chandra and Hubble data, is underway (J. P. Hughes et al. 2010, in preparation). The dense material in the SW (Figure 1) suggests that the forward shock might have recently slowed down. However, we do not find any support for this scenario from the Chandra expansion study, nor from our XMM-Newton/RGS study. Moreover, as the RGS study is skewed toward the bright SW region, it is unlikely that the SW velocity is substantially lower than $6000 \mathrm{~km} \mathrm{~s}^{-1}$.

\section{INTERPRETATION}

The centroid of the broad component in the SW of $-342 \pm$ $28 \mathrm{~km} \mathrm{~s}^{-1}$ indicates the bulk line-of-sight velocity of the shocked 


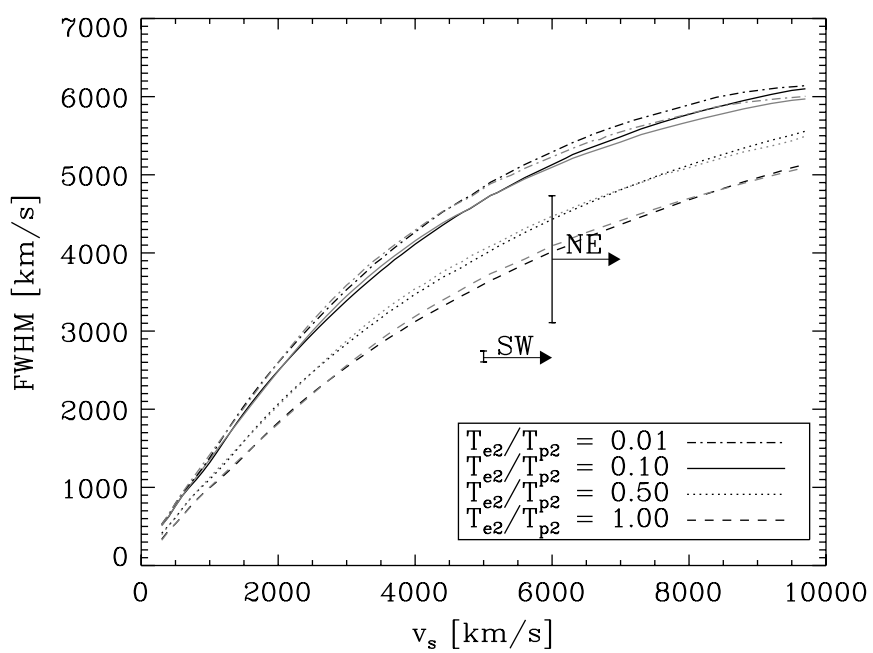

Figure 2. FWHM as a function of $v_{\mathrm{s}}$ for non-accelerating shocks, for different values for $T_{\mathrm{e}} / T_{\mathrm{p}}$ (courtesy of $\mathrm{K}$. Heng \& M. van Adelsberg). The gray (black) lines indicate this relation for plasmas, that are optically thin (thick) to Ly $\beta$ scattering (van Adelsberg et al. 2008). Overplotted is $v_{\mathrm{s}}$ and the measured FWHM for both the SW and NE.

protons. Additionally, the Large Magellanic Cloud (LMC) is moving with $278 \mathrm{~km} \mathrm{~s}^{-1}$ away from us (Richter et al. 1987). This means that we are observing a part of the shell which is moving toward us with $620 \mathrm{~km} \mathrm{~s}^{-1}$ with respect to the LMC. Figure 1 shows a bright, inner shell close to the outer shock. Taking into account the seeing of $0.8-1^{\prime \prime} .0$, our spectrum is probably contaminated by emission from this shell. This part of the shell is likely to dominate the measurement by Ghavamian et al. (2007a), as they also measured a positive offset for the centroid of the broad component.

The flux in the broad component with respect to the flux in the narrow component $\left(I_{\mathrm{b}} / I_{\mathrm{n}}\right)$ declines as a function of shock velocity (Heng \& McCray 2007; van Adelsberg et al. 2008). Our low values for $I_{\mathrm{b}} / I_{\mathrm{n}}$ are therefore consistent with a high shock velocity with likely the highest shock velocity in the NE, which has the lowest $I_{\mathrm{b}} / I_{\mathrm{n}}$. However, as the standard models for interpreting Balmer-dominated shocks do not include the effects of cosmic-ray acceleration, it is not appropriate to use the $I_{\mathrm{b}} / I_{\mathrm{n}}$ values to determine the shock velocities of SNR 0509-67.5.

\subsection{Interpreting the FWHMs}

To determine the cosmic-ray pressure from our measurements, we need to determine $T_{\mathrm{s}, \mathrm{p}}$ from the FWHM. For low temperatures and shock velocities, $k T_{\mathrm{s}, \mathrm{p}}=m_{\mathrm{p}} \sigma^{2}$ (Rybicki \& Lightman 1979), with $\sigma=\mathrm{FWHM} / \sqrt{8 \ln 2}$ in $\mathrm{cm} \mathrm{s}^{-1}$. However, as cross sections for charge exchange decline for high velocities (e.g., Figure 1 in Heng \& McCray 2007), $k T_{\mathrm{s}, \mathrm{p}}>m_{\mathrm{p}} \sigma^{2}$ for higher temperatures and shock velocities. Recent studies focused on determining the FWHM as a function of $v_{\mathrm{s}}$ for nonaccelerating shocks (e.g., Heng \& McCray 2007; van Adelsberg et al. 2008; resulting in Figure 2). Here, we are interested in FWHM as a function of $T_{\mathrm{s}, \mathrm{p}}$ for a given $v_{\mathrm{s}}$ instead. We approximate this concave function linearly between 0,0 and the $\sigma^{2}$ and $T_{\mathrm{s}, \mathrm{p}}$ expected for a non-accelerating shock in thermal equilibrium (Figures 2 and 3 ). In this way, we overestimate $T_{\mathrm{s}, \mathrm{p}}$ and the corresponding thermal pressure, leading to a conservative measure of the cosmic-ray pressure behind the shock front.

We note that the $y$-axis of Figure 5 of van Adelsberg et al. (2008) is labeled incorrectly (M. van Adelsberg 2010, private communication). Instead of showing the "broad H $\alpha$ FWHM,"

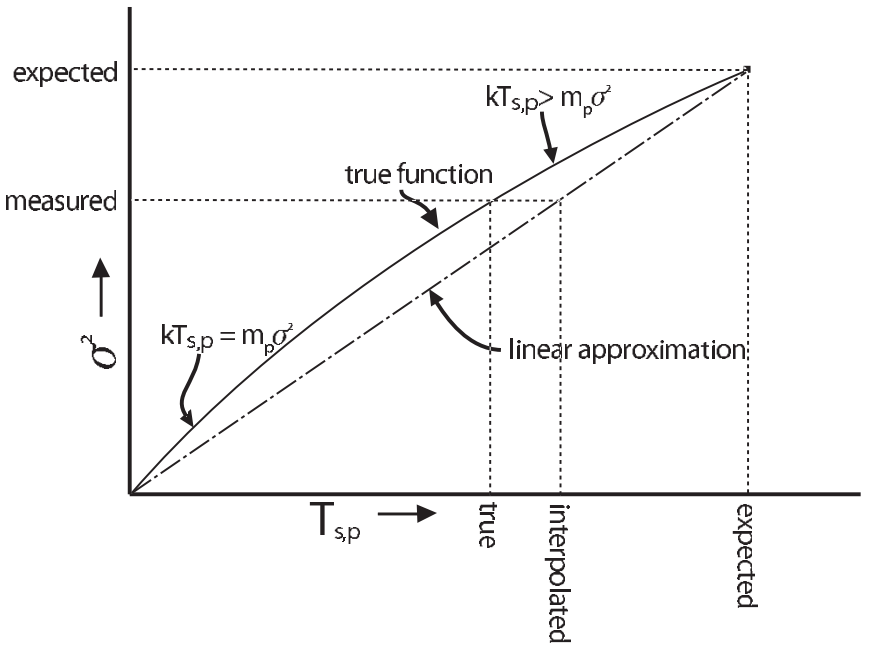

Figure 3. Illustration of $\sigma^{2}$ as a function of $T_{\mathrm{s}, \mathrm{p}}$. "Expected" are the values we expect for a non-accelerating shock (Figure 2). "True" shows the true $T_{\mathrm{s}, \mathrm{p}}$ and "measured" is the corresponding $\sigma^{2}$ we would measure. "Interpolated" shows our estimated $T_{\mathrm{s}, \mathrm{p}}$, based on the measured $\sigma^{2}$. Note that this method leads to an overestimation of $T_{\mathrm{s}, \mathrm{p}}$.

this figure plots the "broad neutral velocity distribution FWHM," which is independent of the emission line considered. To model a specific emission line such as $\mathrm{H} \alpha$, one has to convolve the broad neutral velocity distribution with the relevant atomic cross sections. The FWHM- $v_{\mathrm{s}}$ relations we use in this study, obtained in electronic form from M. van Adelsberg \& K. Heng, are plotted in Figure 2 and are based upon the same relations used to generate Figure 13 and Table 1 of van Adelsberg et al. (2008).

Following Vink (2008a) and Helder et al. (2009), we interpret $T_{\mathrm{s}, \mathrm{p}}$ and $v_{\mathrm{s}}$ in terms of cosmic-ray pressure behind the shock front and cosmic-ray energy flux leaving the system (respectively $w_{\mathrm{CR}}=P_{\mathrm{CR}} / P_{\text {tot }}$ in which $P_{\text {tot }}$ is the total pressure behind the shock front ( $x$-axis in Figure 4$)$ and $\epsilon_{\text {esc }}=F_{\text {esc }} / F_{\text {tot }}$ in which $F_{\text {tot }}$ is the energy flux entering the shock; $\frac{1}{2} \rho_{\mathrm{ISM}} v_{\mathrm{s}}^{3}$ $(y$-axis of Figure 4)). To conservatively estimate the post-shock cosmic-ray pressure, we assume the electrons and ions to be in thermal equilibrium. We add $w_{\mathrm{CR}}$ and $\epsilon_{\mathrm{esc}}$ to the equations of conservation of mass, momentum, and energy over the shock front, which leads to

$$
k T_{\mathrm{s}, \mathrm{p}}=\left(1-w_{\mathrm{CR}}\right) \frac{1}{\chi}\left(1-\frac{1}{\chi}\right) \mu \mathrm{m}_{\mathrm{p}} v_{\mathrm{s}}^{2} .
$$

Formally, $\mu$ is the number-averaged mean particle weight $(\sim 0.54$ for a fully equilibrated and fully ionized plasma with LMC abundances), when considering $k T_{\mathrm{s}, \mathrm{p}}$, we can treat $\mu$ as well as a measure for the temperature equilibration behind the shock front, where $\mu=1$ indicates no temperature equilibration, and $\mu=0.54$ indicates a fully equilibrated plasma. In addition, $\chi$ is the total shock compression ratio. For a non-accelerating, adiabatic shock, $\chi=4$ and hence $k T_{\mathrm{s}, \mathrm{p}}=\frac{3}{16} \mu m_{\mathrm{p}} v_{\mathrm{s}}^{2}$. We define $\beta \equiv k T_{\mathrm{s}, \mathrm{p}} / \frac{3}{16} \mu m_{\mathrm{p}} v_{\mathrm{s}}^{2}$, to characterize the influence of cosmic-ray acceleration on $T_{\mathrm{s}, \mathrm{p}}$. Figure 4 shows $\beta$ in the $\left(w_{\mathrm{CR}}, \epsilon_{\mathrm{esc}}\right)$ frame. The "max" line indicates the ratio of the cosmic-ray pressure and escaping cosmic-ray energy for the most efficiently accelerating shock according to theory (Malkov 1999; Drury et al. 2009). Hence, the hashed region is excluded. 


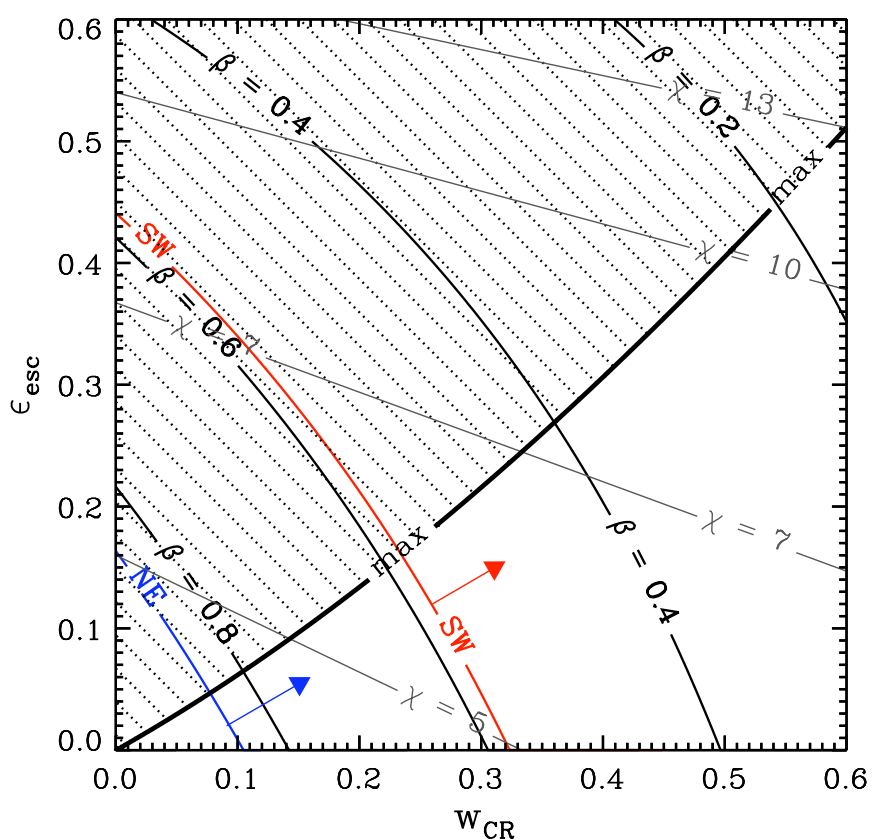

Figure 4. Values for $\beta$ in the $\left(w_{\mathrm{CR}}, \epsilon_{\mathrm{esc}}\right)$ frame. The $\chi$-lines indicate the compression ratio of the plasma behind the shock front. The hashed region requires a shock, more efficient than can be explained with current theoretical models. The red line indicates the lower limit for $\beta$ for the SW. For the NE, $\beta$ is determined assuming $T_{\mathrm{s}, \mathrm{e}} / T_{\mathrm{s}, \mathrm{p}}<0.1$.

\subsection{The Southwest Spectrum}

For $v_{\mathrm{s}}=5000 \mathrm{~km} \mathrm{~s}^{-1}$, we expect to measure at least an FWHM for the broad component of $3600 \mathrm{~km} \mathrm{~s}^{-1}$, corresponding to a $T_{\mathrm{s}, \mathrm{p}}$ of $28.7 \mathrm{keV}$ (Figure 2). This differs significantly from the measured $2680 \pm 70 \mathrm{~km} \mathrm{~s}^{-1}$. We derive a $T_{\mathrm{s}, \mathrm{p}}$ of $15.9 \pm 0.9 \mathrm{keV}$, leading to a $\beta<0.58$, which gives a cosmic-ray pressure behind the shock front of at least $20 \%$ (Figure 4 ).

\subsection{The Northeast Spectrum}

For the NE, the measured FWHM indicates a $v_{\mathrm{s}}=$ $6000 \mathrm{~km} \mathrm{~s}^{-1}$, for $0.2<T_{\mathrm{s}, \mathrm{e}} / T_{\mathrm{s}, \mathrm{p}}<1.0$ (Figure 2). This leads to two possibilities:

1. The shock does not efficiently accelerate cosmic rays and has a $T_{\mathrm{s}, \mathrm{e}} / T_{\mathrm{s}, \mathrm{p}}>0.2$, breaking with the earlier reported trend of $T_{\mathrm{s}, \mathrm{e}} / T_{\mathrm{s}, \mathrm{p}} \propto 1 / v_{\mathrm{s}}^{2}$ for $v_{\mathrm{s}}>400 \mathrm{~km} \mathrm{~s}^{-1}$ (Ghavamian et al. 2007a), as this would give $T_{\mathrm{e}} / T_{\mathrm{p}}<0.01$ for $v_{\mathrm{s}}=6000$ $\mathrm{km} \mathrm{s}^{-1}$.

2. The shock is far out of thermal equilibrium, as we might expect for a fast shock, and accelerates the particles. If we assume $T_{\mathrm{s}, \mathrm{e}} / T_{\mathrm{s}, \mathrm{p}}<0.1$, we expect an FWHM of $>5100 \mathrm{~km} \mathrm{~s}^{-1}$, whereas we measure $3900 \pm 800 \mathrm{~km} \mathrm{~s}^{-1}$. Using the approach from Section 4.1 and Figure 4, we obtain $\beta<0.85$ and hence a cosmic-ray pressure behind the shock front of $>7 \%$ of the total pressure. Note that this is a very conservative lower limit: for both $T_{s, p}$ and $v_{\mathrm{s}}$ we used conservative approximations. Also, at this high shock velocity, the squared line width of the broad component does not increase linearly with $T_{\mathrm{s}, \mathrm{p}}$, but flattens off. This makes the difference in line width lower for a given $\Delta T_{\mathrm{s}, \mathrm{p}}$, which means that the line width is a less sensitive temperature indicator at high shock velocities. Future progress can be made with better shock velocity estimates of the NE region itself, a higher signal-overnoise spectrum of the NE region, and with models for non- radiative Balmer-dominated shocks that include the effects of cosmic-ray acceleration.

A remaining question is whether the magnetic field pressure makes a contribution to the post-shock pressure. As SNR 050967.5 is at a distance of $50 \mathrm{kpc}$, we cannot resolve filaments of Xray synchrotron emission, which are often used for determining post-shock magnetic field strengths (Vink \& Laming 2003). However, Völk et al. (2005) showed that a typical value for the magnetic field pressure is around $3.5 \%$ of the total post-shock pressure. Moreover, according to Bell's theory (Bell 2004) the magnetic field energy density scales as $B^{2} / 8 \pi \sim \frac{1}{2} v_{\mathrm{s}} U_{\mathrm{c}} / c$, with $U_{\mathrm{c}}$ being the cosmic ray energy density. This means that the magnetic field energy density is expected to be about $1 \%$ of the cosmic-ray energy density.

\section{CONCLUSIONS}

We investigated the cosmic-ray acceleration efficiency of the 0509-67.5 SNR in the LMC, by comparing $T_{\mathrm{s}, \mathrm{p}}$, determined from the $\mathrm{H} \alpha$ line widths of the $\mathrm{SW}$ and $\mathrm{NE}$ shocks with shock velocities of respectively $5000 \mathrm{~km} \mathrm{~s}^{-1}$ and $6000 \mathrm{~km} \mathrm{~s}^{-1}$ for the SW and NE, based on X-ray observations. Our study gives the following results.

1. We measured widths of the broad components of the $\mathrm{H} \alpha$ lines in SNR 0509-67.5 to be $2680 \pm 70 \mathrm{~km} \mathrm{~s}^{-1}$ for the SW and $3900 \pm 800 \mathrm{~km} \mathrm{~s}^{-1}$ for the NE.

2. For the SW, we can only explain the line width if we allow for a contribution of $>20 \%$ of the post-shock pressure by cosmic rays.

3. For the NE shock, we have two options: either the shock has a $T_{\mathrm{s}, \mathrm{e}} / T_{\mathrm{s}, \mathrm{p}}>0.2$, breaking with the earlier reported trend of $T_{\mathrm{s}, \mathrm{e}} / T_{\mathrm{s}, \mathrm{p}} \propto 1 / v_{\mathrm{s}}^{2}$ for $v_{\mathrm{s}}>400 \mathrm{~km} \mathrm{~s}^{-1}$ (Ghavamian et al. $2007 \mathrm{a}$ ), or if we assume $T_{\mathrm{s}, \mathrm{e}} / T_{\mathrm{s}, \mathrm{p}}<0.1$, the cosmic-ray pressure behind the shock front is at least $7 \%$ of the total pressure.

This research, together with our previous study (Helder et al. 2009), shows that more than $10 \%$ of the pressure in young SNRs can be contributed by cosmic rays. This is more than the requirement that $10 \%$ of the available energy needs to be in cosmic rays. On the other hand, the cosmic-ray acceleration efficiency may decline for older SNRs, as indicated by a recent study of the Cygnus Loop (Salvesen et al. 2009). So a higher efficiency at a young age may be needed to have an average efficiency of $10 \%$ over the whole lifetime of an SNR.

The authors thank Matthew van Adelsberg and Kevin Heng for kindly providing the data used for Figure 2. We also thank Frank Verbunt for critical reading of the manuscript and Cees Bassa and Peter Jonker for useful discussions on the planning and reduction of optical spectra. This research is based on observations collected with ESO Telescopes at the Paranal Observatory under programme ID 384.D-0518(A) and on observations obtained with the XMM-Newton and Chandra satellites. E.A.H. and J.V. are supported by the Vidi grant of J.V. from the Netherlands Organization for Scientific Research (NWO). D.K. is supported by an "open competition" grant from NWO. This research has made use of the NASA/IPAC Extragalactic Database (NED) which is operated by the Jet Propulsion Laboratory, California Institute of Technology, under contract with the National Aeronautics and Space Administration. 


\section{REFERENCES}

Abdo, A. A., et al. 2010, ApJ, 710, L92

Appenzeller, I., et al. 1998, Messenger, 94, 1

Badenes, C., Hughes, J. P., Cassam-Chenaï, G., \& Bravo, E. 2008, ApJ, 680, 1149

Bell, A. R. 2004, MNRAS, 353, 550

Chevalier, R. A., Kirshner, R. P., \& Raymond, J. C. 1980, ApJ, 235, 186

Drury, L. O'C., Aharonian, F. A., Malyshev, D., \& Gabici, S. 2009, A\&A, 496, 1

Ellison, D. C., Decourchelle, A., \& Ballet, J. 2004, A\&A, 413, 189

Ellison, D. C., Patnaude, D. J., Slane, P., \& Raymond, J. 2010, ApJ, 712, 287

Ghavamian, P., Blair, W. P., Sankrit, R., Raymond, J. C., \& Hughes, J. P. 2007a, ApJ, 664, 304

Ghavamian, P., Laming, J. M., \& Rakowski, C. E. 2007b, ApJ, 654, L69

Hamuy, M., Suntzeff, N. B., Heathcote, S. R., Walker, A. R., Gigoux, P., \& Phillips, M. M. 1994, PASP, 106, 566

Helder, E. A., et al. 2009, Science, 325, 719

Heng, K. 2009, PASA, 27, 23

Heng, K., \& McCray, R. 2007, ApJ, 654, 923

Hughes, J. P., et al. 1995, ApJ, 444, L81

Kosenko, D. I. 2006, MNRAS, 369, 1407

Kosenko, D., Helder, E. A., \& Vink, J. 2010, A\&A, in press (arXiv:1001.0983)

Kosenko, D., Vink, J., Blinnikov, S., \& Rasmussen, A. 2008, A\&A, 490, 223

Lee, J.-J., Koo, B.-C., Raymond, J., Ghavamian, P., Pyo, T.-S., Tajitsu, A., \& Hayashi, M. 2007, ApJ, 659, L133

Malkov, M. A. 1999, ApJ, 511, L53
Malkov, M. A., \& Drury, L. 2001, Rep. Prog. Phys., 64, 429

Osterbrock, D. E., Fulbright, J. P., Martel, A. R., Keane, M. J., Trager, S. C., \& Basri, G. 1996, PASP, 108, 277

Rest, A., Matheson, T., \& Blondin, S., et al. 2008, ApJ, 680, 1137

Richter, O., Tammann, G. A., \& Huchtmeier, W. K. 1987, A\&A, 171, 33

Rybicki, G. B., \& Lightman, A. P. 1979, Radiative Processes in Astrophysics (New York: Wiley-Interscience)

Salvesen, G., Raymond, J. C., \& Edgar, R. J. 2009, ApJ, 702, 327

Smith, R. C., Kirshner, R. P., Blair, W. P., \& Winkler, P. F. 1991, ApJ, 375, 652

Sorokina, E. I., Blinnikov, S. I., Kosenko, D. I., \& Lundqvist, P. 2004, Astron. Lett., 30, 737

Truelove, J. K., \& McKee, C. F. 1999, ApJS, 120, 299

Tuohy, I. R., Dopita, M. A., Mathewson, D. S., Long, K. S., \& Helfand, D. J. 1982, ApJ, 261, 473

van Adelsberg, M., Heng, K., McCray, R., \& Raymond, J. C. 2008, ApJ, 689, 1089

Vink, J. 2008a, in AIP Conf. Proc. 1085, 4th International Meeting on High Energy Gamma-Ray Astronomy, ed. F. A. Aharonian, W. Hofmann, \& F. Rieger (Melville, NY: AIP), 69

Vink, J. 2008b, ApJ, 689, 231

Vink, J., Bleeker, J., van der Heyden, K., Bykov, A., Bamba, A., \& Yamazaki, R. 2006, ApJ, 648, L33

Vink, J., \& Laming, J. M. 2003, ApJ, 584, 758

Völk, H. J., Berezhko, E. G., \& Ksenofontov, L. T. 2005, A\&A, 433, 229

Warren, J. S., \& Hughes, J. P. 2004, ApJ, 608, 261

Warren, J. S., et al. 2005, ApJ, 634, 376 